\title{
DESIGUALDADE DE OPORTUNIDADES NO BRASIL: EFEITOS DIRETOS E INDIRETOS
}

\author{
Erik Alencar de Figueiredo* \\ Cleiton Roberto da Fonseca Silva ${ }^{\dagger}$ \\ Herbert de Oliveira Rego ${ }^{\ddagger}$
}

\begin{abstract}
Resumo
O objetivo deste estudo é mensurar a desigualdade de oportunidades brasileira. Para tanto, utilizam-se a abordagem teórica desenvolvida por Pistolesi (2009) e o método de geração de contrafactuais de Chernozhukov et al. (2009). A estratégia empírica adotada permite ao menos dois avanços em relação às propostas pregressas: a) admite o efeito da variável explicativa em diferentes pontos da variável dependente e b) emprega um método que generaliza diversas abordagens utilizadas na literatura. Os resultados indicam que a proporção da desigualdade de oportunidades na desigualdade total é de cerca de $35 \%$.
\end{abstract}

Palavras-chave: Desigualdade de oportunidades; Método contrafactual.

\begin{abstract}
The aim of this study is to measure the inequality of opportunities in Brazil. For this purpose, we use the theoretical approach developed by Pistolesi (2009) and an empirical strategy based on combining the method of counterfactual generation by Chernozhukov, Fernández-Val e Melly (2009). The results indicate that the proportion of inequality of opportunity in total Brazilian inequality is $35 \%$.
\end{abstract}

Keywords: Inequality of opportunity; Quantile regressions; Counterfactual method.

JEL classification: C22, E31

\footnotetext{
* Programa de Pós-Graduação em Economia. Universidade Federal daParaíba, Brasil. Pesquisador do CNPq. E-mail: eafigueiredo@gmail.com

† Programa de Pós-Graduação em Economia. Universidade Federal daParaíba, Brasil. E-mail: cleiton.roberto@yahoo.com.br

‡ Departamento de Finanças e Contabilidade. Universidade Federal da Paraíba, Brasil. E-mail: hebert.rgo@gmail.com
} 


\section{Introdução}

A estabilização da inflação e a manutenção de um contexto macroeconômico favorável a partir da segunda metade da década de 1990 promoveram as bases para o avanço da discussão sobre distribuição de renda no Brasil. Políticas de transferência foram implementadas e a desigualdade, crescente no período de 1960-1990, passou a declinar após 1995 (Azevedo 2007, Figueiredo \& Ziegelmann 2009), em concomitância com uma melhora nos níveis de pobreza e indigência (Ramos \& Santana 2003). Contudo, mesmo diante desse cenário, o Brasil continua apresentando uma das piores iniquidades de renda do mundo (cf. (IPEA 2006)).

Diante desse cenário, a literatura especializada passou a questionar os possíveis determinantes dessa desigualdade. As abordagens são diversas e vão desde a captação dos baixos níveis de mobilidades econômica e social, em especial, os resultados referentes às mobilidades de renda inter e intrageracional, contidos, respectivamente, em Ferreira \& Veloso (2006), Figueiredo \& Ziegelmann (2010a), e de mobilidade social exposta em Pastore \& Silva (1999), até a detecção do elevado nível de desigualdade de oportunidades brasileiro presente em Bourguignon et al. (2007).

Focando no conceito de desigualdade de oportunidades, as investigações admitem que os resultados econômicos dos indivíduos são determinados tanto por variáveis de esforço (fatores de responsabilidade), quanto por variáveis de circunstâncias que fogem do controle dos agentes econômicos (fatores de não responsabilidade). Para tanto, é necessário decompor a desigualdade a partir da criação de duas distribuições contrafactuais onde se equaliza a desigualdade resultante de variáveis de esforço (abordagem direta) e de circunstância (abordagem indireta).

Normalmente, a decomposição contrafactual pode ser obtida por duas vias, que se diferenciam a partir do tratamento dispensado a variável de esforço. Bourguignon et al. (2007), por exemplo, a considera um fator observável. Já a abordagem recente de Pistolesi (2009) a toma como não observável. Nesse último caso, a construção das distribuições contrafactuais admite a seguinte suposição: duas pessoas com diferentes níveis de oportunidade, mas no mesmo percentil da sua distribuição condicionada ao seu tipo, empreendam o mesmo nível de esforço. Essa condição é reconhecida como Roemer's Identification Axiom - RIA (Roemer 1996) ${ }^{1}$.

Apesar de adotar essa forte suposição teórica, a proposta contida em Pistolesi (2009) é relevante ao admitir o efeito das variáveis explicativas em diferentes pontos da variável dependente e ao adotar a construção das distribuições contrafactuais a partir da consideração dos fatores de responsabilidade e não responsabilidade. No entanto, seu processo de geração dos contrafactuais é alvo de críticas na literatura. O autor utiliza um modelo semi paramétrico sugerido por Donald et al. (2000) onde se considera que os coeficientes associados às covariáveis se relacionam de forma discreta com a variável dependente, o que constitui uma importante restrição para o processo de inferência.

Nesse contexto, um avanço empírico importante pode ser alcançado a partir do método de geração dos contrafactuais apresentado por Chernozhukov et al. (2009). Esse ferramental constitui uma generalização para diversas abordagens, inclusive a proposta por Donald et al. (2000). Esse método mais amplo

\footnotetext{
${ }^{1}$ Vantagens e desvantagens desses métodos serão discutidos na seção seguinte.
} 
permite uma melhor captação dos efeitos das covariáveis a partir da utilização de estimadores que possuem distribuição assintótica bem definida.

Dito isso, o objetivo deste artigo é mensurar a desigualdade de oportunidades brasileira. Para tanto, serão adotadas a abordagem teórica proposta por Pistolesi (2009) e o método de geração dos contrafactuais proposto por Chernozhukov et al. (2009) . O banco de dados segue a PNAD 1996 devido ao suplemento de mobilidade social disponível.

Por fim, o estudo está dividido da seguinte maneira: na próxima seção serão detalhados aspectos teóricos e empíricos em uma breve revisão de literatura. A seção 3 apresenta a estratégia empírica, incluindo os dados, a geração dos contrafactuais e a mensuração da desigualdade de oportunidades. Os resultados são apresentados na seção 4 . Por fim, seguem as considerações finais e as referências.

\section{Uma Breve Leitura Teórica e Empírica}

Dois conceitos de desigualdade de oportunidades têm prevalecido na literatura. O primeiro assume que a desigualdade de renda entre os indivíduos é originada por fatores de responsabilidade e não responsabilidade (Roemer 1998). Em outras palavras, parte da renda é determinada por variáveis de esforço como nível educacional, decisão de migrar, horas trabalhadas por ano, entre outras; e a outra por fatores que fogem do controle dos agentes econômicos, variáveis circunstância, ou seja, background familiar (nível educacional e ocupação dos pais), atributos individuais como raça, gênero, idade ou região de nascimento, entre outras. Uma segunda abordagem foca no conjunto de oportunidades disponível ${ }^{2}$, comparando se os indivíduos com diferentes conjuntos de oportunidades possuem vantagens entre si.

A adoção da abordagem proposta, inicialmente, por Roemer (1998), entende que somente a desigualdade oriunda das variáveis de circunstância é socialmente indesejável ${ }^{3}$. Sob esse enfoque, pode-se decompor a iniquidade de uma determinada distribuição de renda, $I\left(Y^{a}\right)^{4}$, a partir da criação de duas distribuições contrafactuais. Uma livre da desigualdade relacionada às circunstâncias, $I\left(Y^{c}\right)$, e a outra onde não há desigualdade resultante do esforço, $I\left(Y^{e}\right)$. Diante disso, a desigualdade de oportunidades pode ser mensurada a partir de dois indicadores: no primeiro se considera a diferença entre as desigualdades da distribuição atual e da distribuição isenta de diferenciais de circunstâncias $I\left(Y^{a}\right)-I\left(Y^{c}\right)$ e; o segundo sumarizando a desigualdade oriunda apenas dos fatores de circunstância, $I\left(Y^{e}\right)$. No mais, os estudos que optam pela estimação das distribuições contrafactuais costumam ser classificados em dois grupos: os que assumem a variável de esforço como um fator observável e os que a consideram um fator não-observável.

Dentro do primeiro grupo, destaca-se o paper influente de Bourguignon et al. (2007). Analisando a desigualdade de oportunidades brasileira, os autores propõem uma abordagem paramétrica onde os rendimentos, $Y_{i}$, são explicados pelas variáveis de circunstância $\left(C_{i}\right)$ e de esforço $\left(E_{i}\right)$, ou seja:

\footnotetext{
${ }^{2}$ Para uma comparação entre as abordagens, ver Ooghe et al. (2007).

${ }^{3}$ Nesse ponto há um importante avanço em relação as teorias tradicionais como, por exemplo, a de Atkinson (1970), onde toda a desigualdade é socialmente indesejável.

${ }^{4}$ Sendo I um índice de desigualdade como o Gini ou Theil, por exemplo.
} 


$$
\ln Y=\alpha C_{i}+\lambda E_{i}+u_{i}
$$

e, devido a endogeneidade das circunstâncias:

$$
E_{i}=\Gamma C_{i}+v_{i}
$$

Onde $\alpha$ e $\lambda$ são vetores de parâmetros, $\Gamma$ representa uma matriz de coeficientes associados as variáveis de circunstância e de esforço e $u_{i}$ e $v_{i}$ são fatores aleatórios, inclusive a sorte. Dado que muitas variáveis de circunstância e esforço são não observáveis ou de difícil mensuração, os parâmetros das equações de rendimento estão sujeitas a vieses. Cientes disso, o estudo promove uma simulação de Monte Carlo, obtendo intervalos para variação para os coeficientes estimados. O procedimento é baseado no "monotone treatment response" fornecido por Manski \& Pepper (2000).

Seus resultados sugerem que as variáveis de circunstância correspondem a cerca de $23 \%$ da desigualdade total, onde, $60 \%$ são efeitos captados em 1 e $40 \%$ se dão a partir de 2 . O backgroud familiar se apresenta como o principal fator de determinação do nível de oportunidades, dado que cerca de $70 \%$ do efeito das circunstâncias se deve a escolaridade do pai ${ }^{5}$.

Conforme exposto, considerar a variável de esforço como um fator não observável implica em admitir o Roemer's Identification Axiom (Roemer 1996). Dentro desse segundo enfoque de investigação, destaca-se o estudo de Checchi \& Peragine (2009). Em sua estratégia empírica, os autores dividem a população em $n$ tipos e $m$ percentis (ou tranches), a partir da criação de uma matriz $M^{a}$ de dimensão $n \times m$, representando a distribuição de renda vigente. Essa estrutura faz com que cada combinação do tipo com o tranche, corresponda a um nível de renda. Como supõe-se que em cada tranche o nível de esforço é o mesmo, a distribuição contrafactual é obtida a partir da substituição das colunas da matriz original por colunas onde vigora um valor médio para a renda do tranche. Dessa forma, a nova matriz, $M^{c}$, não conterá diferenças de renda resultante das circunstâncias. Por conseguinte, o indicador para a desigualdade de oportunidades é calculado a partir de: $\left.\left.I(M)^{a}\right)-I(M)^{c}\right)$. Ou seja, novamente equalizam-se os efeitos das circunstâncias.

A aplicação dessa estratégia aos dados italianos, no período de 1993 a 2000, divididos em cinco tipos e vinte tranches, indicaram que a Região Sul do país apresenta o dobro da desigualdade de oportunidades da Região Centro-Norte. A comparação desses resultados com os obtidos por Bourguignon et al. (2007), reforçam a magnitude da desigualdade de oportunidades brasileira. Como já destacado, no Brasil a proporção da desigualdade de oportunidades na desigualdade total é de cerca de $23 \%$. Na Itália essa proporção é de cerca de $10 \%$.

Alternativamente, a desigualdade de oportunidades pode ser mensurada a partir da comparação de distribuições condicionadas. Nesse caso, como destacado no início da seção, o foco é direcionado para o conjunto de oportunidades ao qual os indivíduos estão inseridos. Essa visão foi proposta, inicialmente, por Pattanaik \& Xu (1990) e Kranich (1996) e desenvolvida, posteriormente, por Lefranc et al. (2004). A idéia é simples: a igualdade de oportunidades prevalece se e somente se os indivíduos associados a um particular conjunto

\footnotetext{
${ }^{5}$ Esses resultados são compatíveis com os achados da literatura relacionada a mobilidade intergeracional brasileira, em especial, Ferreira \& Veloso (2006).
} 
de circunstâncias, não apresentarem vantagens em relação a um outro grupo de indivíduos pertencentes a um outro conjunto de circunstâncias.

Baseado nesse princípio, Lefranc et al. (2008) promovem uma comparação internacional envolvendo nove países desenvolvidos. Para tanto, consideramse duas variáveis de circunstância, educação e ocupação dos pais, divididas em três categorias. Visando estabelecer a significância estatística das diferenças nas distribuições condicionadas, os autores adotam uma série de testes para dominância estocástica. Seus resultados apontam para a existência de desigualdade de oportunidades na França, Itália e EUA. Sendo a Suécia a única nação onde vigora a igualdade de oportunidades. Essas evidências são corroboradas a partir do cálculo de um índice de desigualdade de oportunidades.

Já o estudo de O'Neil et al. (2001) propõe um modelo teórico onde se estabelece uma associação entre a distribuição condicionada e o conjunto de oportunidades dos indivíduos. Para tanto, os autores sugerem uma estratégia empírica não-paramétrica baseada em estimadores double-kernel com multiplestep procedure. Entretanto, Figueiredo \& Ziegelmann (2010b) afirmam que "a drawback of such methods is that they do not guarantee that the estimated functions are non-decreasing and restricted". Sugerindo uma estratégia robusta para a estimação das distribuições condicionadas, Figueiredo \& Ziegelmann (2010b) observam que no caso dos EUA a diferença de esforço entre os indivíduos pertencentes aos percentis 0,25 e 0,75 é de aproximadamente $56 \%$. Para o Brasil essa diferença chega a $112 \%{ }^{6}$.

Recentemente, Pistolesi (2009) propôs um método alternativo para a captação da desigualdade de oportunidades. Sua abordagem incondicional é similar às estratégias de Bourguignon et al. (2007) e Checchi \& Peragine (2009), avançando em, pelo menos, dois sentidos: a) ao captar os efeitos das variáveis explicativas em diferentes pontos da distribuição da variável dependente e; b) ao sugerir duas medidas de desigualdade de oportunidades, a primeira captando a desigualdade referente ao esforço (a partir da equalização das circunstâncias) e a segunda mensurando a desigualdade referente às circunstância (eliminando o efeito dos fatores de esforço).

Entretanto, apesar do seu procedimento apresentar vantagens em relação às abordagens anteriores, o que justifica a sua adoção, ele não está isento de críticas, principalmente no que se refere ao processo de estimação das distribuições contrafactuais ${ }^{7}$. Conforme comentado, o autor utiliza o modelo semi-paramétrico sugerido por Donald et al. (2000). Nele se considera que os coeficientes associados às covariáveis se associam de forma discreta com a variável dependente. De acordo com Machado \& Mata (2000), essa relação constitui uma restrição importante para o processo de inferência. No mais, questões relacionadas a omissão de variáveis relevantes não são abordadas.

Diante dessas informações, a seção seguinte apresentará uma alternativa ao método de estimação usado em Pistolesi (2009). Em primeiro lugar, subseção 3.1, é destacada a estrutura básica utilizada para obter as medidas direta e indireta da desigualdade de oportunidades. Em seguida, apresenta-se o método contrafactual desenvolvido por Chernozhukov et al. (2009), destacando

\footnotetext{
${ }^{6}$ Outros indicadores revelam o elevado nível de desigualdade de oportunidades brasileiro. Ver Cogneau \& Gignoux (2005) and Barros et al. (2009).

${ }^{7}$ É evidente que todas as abordagens apresentadas estão sujeitas a importantes críticas e vieses resultantes, principalmente, da existência de endogeneidade, da má especificação das formas funcionais da equação de rendimento ou da adoção do RIA. Para uma leitura mais detalhada desses métodos, ver Ramos \& Van de gaer (2009).
} 
que esse ferramental constitui uma generalização para a abordagem de Donald et al. (2000).

\section{Estratégia Empírica}

\subsection{Mensurando a Desigualdade de Oportunidades}

De acordo com Pistolesi (2009), a densidade para os rendimentos, $Y$, pode ser expressa por:

$$
f(Y)=\int_{X^{e}} \int_{X^{c}} F_{Y}\left(Y \mid X^{e}, X^{c}\right) H_{X^{e}}\left(X^{e} \mid X^{c}\right) G\left(X^{c}\right) d X^{e} d X^{c}
$$

onde $X^{e}$ e $X^{c}$ representam, respectivamente, as variáveis de esforço e circunstância, $F_{Y}(Y \mid \cdot)$ é a distribuição condicionada da renda, $H_{X^{e}}(\cdot \mid)$ é a distribuição condicionada do esforço e $G(\cdot)$ é distribuição marginal das cincunstâncias.

Com essa definição, assume-se que um indivíduo exerce um maior esforço relativo, se ele estiver ranqueado em um ponto mais alto de sua distribuição condicionada. Em outras palavras, pessoas com diferentes níveis de circunstância, mas pertencentes ao mesmo quantil de sua distribuição, exercem o mesmo nível de esforço.

Baseado em 3, a autor sugere duas formas de mensurar a desigualdade de oportunidades. Na primeira, abordagem indireta, a igualdade de oportunidades é definida como a situação onde as diferenças de renda são oriundas somente do diferencial do esforço. Na segunda, abordagem direta, a igualdade de oportunidades prevalecerá se e somente se os indivíduos com diferentes níveis de circunstância, porém, exercendo a mesma quantidade de esforço, possuirem o mesmo nível de renda.

\section{Abordagem Indireta}

Para adotar a abordagem indireta é necessário gerar uma distribuição contrafactual para o esforço condicionado. Sendo assim, $H_{X^{e}}\left(\cdot \mid X^{c}\right)$ será simulada considerando um valor fixo para as circunstâncias, digamos $\bar{X}^{c}$, resultando em:

$$
\hat{H}_{X^{e}}\left(\cdot \mid \bar{X}^{c}\right) .
$$

Feito isso, gera-se um nível individual de esforço simulado. Para tanto, é necessário preservar as diferenças nos níveis de esforço relativo, ou seja, o nível de esforço é gerado no mesmo quantil de esforço da distribuição original. Para tanto, calcula-se, para cada observação $i, \tilde{X}_{i}^{e}=\hat{H}_{X^{e}}^{-1}\left(H_{X^{e}}\left(X_{i}^{e} \mid X^{c}\right) \mid \bar{X}^{c}\right)$, com $H_{X^{e}}(\cdot)$ representando a Conditional Distribution Function (CDF) do esforço observado e $\hat{H}_{e}(\cdot)$ a CDF do esforço simulado.

No segundo passo, estima-se a distribuição condicionada da renda, considerando o esforço simulado e o valor fixo para as circunstâncias $\hat{F}_{Y}=Y \mid \tilde{X}^{e}, \bar{X}^{c}$ ). Por fim, essas duas etapas geram:

$$
\hat{f}(Y)=\int_{\tilde{X}^{e}} \hat{F}_{Y}\left(Y \mid \tilde{X}^{e}, \bar{X}^{c}\right) \hat{H}_{X^{e}}\left(\tilde{X}^{e} \mid \bar{X}^{c}\right) d \tilde{X}^{e} .
$$


Essa distribuição provê uma medida indireta para a desigualdade de oportunidades, onde as desigualdades serão fruto apenas dos diferenciais de esforço.

\section{Abordagem Direta}

A abordagem direta é similar a indireta, mudando a variável utilizada na geração da contrafactual e o tratamento dado na geração do esforço simulado. Dessa forma, o nível de esforço é fixado, em geral pela média, resultando em $\tilde{\tilde{X}}^{e}=\hat{\hat{H}}_{X^{e}}^{-1}\left(H_{X^{e}}\left(\bar{X}^{e} \mid X^{c}\right) \mid X^{c}\right)$. Esse procedimento apaga os diferenciais de esforço within group, preservando a desigualdades de esforço between group. Assim, a distribuição condicionada da renda, considerando apenas os diferencias de circunstâncias, será: $\hat{\hat{F}}_{Y}=\left(Y \mid X^{c}, \bar{X}^{e}\right)$. Consequentemente, a distribuição incondicionada da renda, que reflete apenas os diferenças nas circunstâncias será:

$$
\hat{\hat{f}}(Y)=\int_{X^{c}} \int_{\bar{X}^{e}} \hat{\hat{F}}_{Y}\left(Y \mid \bar{X}^{e}, X^{c}\right) \hat{\hat{H}}_{X^{e}}\left(\bar{X}^{e} \mid X^{c}\right) G\left(X^{c}\right) d \bar{X}^{e} d X^{c} .
$$

Os indicadores 6 e 5 são medidas simétricas para a desigualdade de oportunidades. A obtenção dessas densidades requer o cálculo de distribuições condicionadas para o esforço e a renda.

\subsection{Método Contrafactual}

O objetivo desta subseção é sumarizar o método de inferência de distribuições contrafactuais proposto por Chernozhukov et al. (2009). Serão destacadas as relações entre a variável de interesse e as covariáveis antes e depois de uma eventual política, e alguns funcionais resultantes. Por fim, discutem-se os métodos utilizados no processo de inferência, com destaque especial para a generalização dos estimadores de Donald et al. (2000).

\section{Resultados Observados e Contrafactuais}

A escolha do ferramental desenvolvido por Chernozhukov et al. (2009) é justificavél por constituir um importante avanço em relação aos métodos regressionbased pregressos. O principal deles é o desenvolvimento das teorias de distribuição e inferência para os estimadores. No mais, os estimadores podem se basear em uma classe ampla de métodos de regressão para as distribuições condicionais, tais como, as regressões clássica e quantílica, os modelos de duração e as distribution regressions. Dessa forma, os autores incluem intrumentais anteriores, como os de Gosling et al. (2000), Donald et al. (2000) e Machado \& Mata (2000), como casos especiais da sua teoria ${ }^{8}$.

Nessa abordagem, a população investigada é dividida em dois grupos indexados por $j \in\{0,1\}$. O grupo de referência é representado por $j=0$, enquanto que o grupo $j=1$ sumariza os resultados contrafactuais. Para estabelecer a análise de regressão, é importante definir a distribuição condicional (u-quantile) de $Y$ dado $X$ no grupo $j, Q_{Y_{j}}(u \mid x)$, e a distribuição marginal para

\footnotetext{
${ }^{8}$ É importante destacar que esta análise se concentra apenas nos métodos regression-based, em detrimento a literatura relacionada aos métodos propensity score (ver DiNardo et al. (1996) e Firpo et al. (2007), entre outros). Uma comparação entre os métodos de regressão e de propensity score pode ser encontrada no capítulo 3 de Angrist \& Pischke (2008).
} 
o $p$-vetor das covariadas $X$ no grupo $k, F_{X_{k}}$, para $j, k \in\{0,1\}$. Em seguida, define-se a variável de interesse $Y_{j}^{j}$ como uma função das covariáveis e de um distúrbio não-aditivo $U_{j}^{j}$. Considerando a representação de Skorohod, essa relação será:

$$
Y_{j}^{j}=Q_{Y_{j}}\left(U_{j}^{j} \mid X_{j}\right)
$$

onde $U_{j}^{j} \sim U(0,1)$ independente de $X_{j} \sim F_{X_{j}}$, para $j \in\{0,1\}^{9}$.

$\mathrm{O}$ experimento contrafactual adotado nesse estudo, consiste em gerar o resultado da conditional quantile function para o grupo $j=1, Q_{Y_{1}}(u \mid x)$, mantendo as distribuições marginais das covariáveis do grupo $j=0$, ou seja, $X_{0} \sim$ $F_{X_{0}}$. Sendo assim, define-se o resultado contrafactual $Y_{1}^{0}$ por:

$$
Y_{1}^{0}:=Q_{Y_{1}}\left(U_{1}^{0} \mid X_{0}\right)
$$

onde $U_{1}^{0} \sim U(0,1)$ independente de $X_{0} \sim F_{X_{0}}$.

A obtenção dos parâmetros de interesse, em especial o índice de desigualdade, requer a definição de algumas funções específicas, como a distribuição condicional associada a função quantílica $Q_{Y_{j}}(u \mid x)$,

$$
F_{Y_{j}}(y \mid x)=\int_{0}^{1} I\left\{Q_{Y_{j}}(u \mid x) \leq y\right\} d u, \quad j \in\{0,1\}
$$

onde $I\{\cdot\}$ é uma função indicadora que assume valor 1 se o argumento é verdadeiro e 0 caso contrário e; dada a definição 8 , a distribuição marginal de interesse:

$$
F_{Y_{j}}^{k}(y):=\operatorname{Pr}\left\{Y_{j}^{k} \leq y\right\}=\int_{X} F_{Y_{j}}(y \mid x) d F_{X_{k}}(x), j, k \in\{0,1\}
$$

Diante disso, o cálculo da curva de Lorenz, por exemplo, pode ser efetuado por:

$$
L\left(y, F_{Y_{j}}^{k}\right)=\frac{\int_{0}^{y} t d F_{Y_{j}}^{k}(t)}{\int_{0}^{\infty} t d F_{Y_{j}}^{k}(t)}
$$

\section{Inferência}

Os resultados da subseção anterior destacam a importância da função quantílica condicionada e da função de distribuição condicionada para a estimação dos contrafactuais. A partir de uma série de exemplos, Chernozhukov et al. (2009) demonstram que a modelagem e a estimação dessas funções condicionadas podem ser efetuadas a partir de uma série de métodos conhecidos na literatura, inclusive vários modelos de tratamento endógenos ou exógenos. ${ }^{10}$

Como já mencionado, a análise contrafactual adotada por Pistolesi (2009), baseia-se nos modelos de sobrevivência construidos por Donald et al. (2000),

\footnotetext{
${ }^{9}$ Diferente dos modelos de regressão clássicos, a representação de Shokohod não necessita da hipótese da relação aditiva entre os erros e as covariáveis. Para detalhes, ver Van der Vaart \& Wellner (1996).

${ }^{10}$ Convém ressaltar que a ausência de instrumentos apropriados impossibilitam a adoção de estratégias que envolvam a utilização de variáveis instrumentais, tais como as listadas em Giustinelli (2010).
} 
onde a função condicionada é modelada por: $F_{Y}(y \mid x)=\exp (\exp (m(x)+t(y)))$. Segundo Chernozhukov et al. (2009), esta estrutura apresenta uma importante limitação na modelagem das covariáveis, dado que $t(y)$ limita-se a representação location-shift:

$$
t(Y)=m(X)+V
$$

onde $V$ é independente de $X$.

Contudo, esses modelos podem ser entendidos como um caso particular de uma classe mais ampla de estimadores, denominada de distribution regression que, por possuirem um caráter geral, são mais flexíveis na captação dos efeitos das covariáveis. Para tanto, a $F_{Y}(y \mid x)$ é modelada, para cada threshold $y$, a partir de

$$
F_{Y}(y \mid x)=\Lambda(m(y, x)),
$$

onde $\Lambda$ é conhecida como link function e $m(y, x)$ é irrestrita em $y$. Note que os modelos de duração são um caso particular de 13 quando $\Lambda(v)=$ $\exp (\exp (v))$ e $m(y, x)=m(x)+t(y)$. Seguindo Han \& Hausman (1990), $\Lambda$ pode assumir uma especificação probit ou logit e $m(y, x)=x^{\prime} \beta$, onde, $\beta$ é uma função desconhecida de $y$. A estimação é realizada por máxima verossimilhança com base no momento: ${ }^{11}$

$$
G(W, \beta, y)=\frac{\Lambda\left(X^{\prime} \beta\right)-I\{Y \leq y\}}{\Lambda\left(X^{\prime} \beta\right)\left(1-\Lambda\left(X^{\prime} \beta\right)\right)} \lambda\left(X^{\prime} \beta\right) X,
$$

onde $\lambda$ é derivada de $\Lambda$.

As distribuições assintóticas para os estimadores são demonstradas em Chernozhukov et al. (2009). Em resumo, observa-se que para uma $F_{Y}(y \mid x)=$ $\Lambda\left(x^{\prime} \beta\right)$, com $\Lambda$ correspondendo a uma distribuição logistica ou normal, as estimativas de máxima verossimilhança para $\beta$ e para a $F_{Y}(y \mid x)$ obedecem o functional central limit theorem.

\subsection{Dados}

Os dados utilizados neste estudo são parecidos com os do Bourguignon et al. (2007). Tratam-se de informações captadas na Pesquisa Nacional por Amostras de Domicílios (PNAD) para o ano de 1996. Este ano foi escolhido por conta do suplemento de mobilidade social, onde são sumarizadas as informações relacionadas à educação, escolaridade e ocupação dos pais dos indivíduos selecionados. Consideraram-se somente os homens chefes de família, com idade entre 25 e 65 anos, residindo em zonas urbanas. Os rendimentos são representados pelo salário por hora ${ }^{12}$, expresso em Reais de setembro de 2007.

O vetor de variáveis de circunstâncias inclui: a) uma dummie para raça, que assume valor 1 para os não-brancos (i.e. negros, pardos e indígenas) e 0 para os brancos (brancos e asiáticos); b) uma dummie para as regiões, que assume valor 1 se o indivíduo reside nas Regiões mais dinâmicas do país, Sul,

\footnotetext{
${ }^{11}$ Alternativamente, (13) pode ser inferida por estimadores de regressão quantílica, considerando a especificação $Q_{Y}(U \mid X)=m^{-1}\left(\Lambda^{-1}(U), X\right)$, onde, $U \sim U(0,1)$ independente de $X$.

${ }^{12}$ Renda de todos os trabalhos dividida pelo número de horas trabalhadas.
} 
Sudeste e Centro-Oeste e 0 caso contrário (Regiões Norte e Nordeste); c) educação do pai e da mãe, expresas em anos de estudo ${ }^{13}$, e; d) a posição ocupacional do pai, dividida nas seis categorias propostas por Pastore \& Silva $(1999)^{14}$.

As variáveis de esforço são representadas por: a) os anos de estudo do indivíduo; b) uma dummie para migração ${ }^{15} \mathrm{e}$; c) o seu status no mercado de trabalho (trabalhador formal, sem carteira de trabalho assinada ou trabalhando por conta própria). Consideram-se ainda dummies para os coortes, onde, o primeiro coorte representa os indivíduos nascidos entre 1936 e 1940 (variável dummie $C_{1}$ ); o segundo os que nasceram entre 1941 e 1945 e assim por diante até o último coorte, contendo aqueles que nasceram entre 1966 e 1970 (variável dummie $C_{7}$ ).

A amostra conta com 24.293 indivíduos. Ao considerar o desenho complexo, ela é expandida para 19.565.783 indivíduos. Algumas estatísticas descritivas são apresentadas na Tabela 1 , onde, as informações estão divididas em variáveis de esforço e circunstâncias. O primeiro destaque é o maior nível educacional médio dos filhos, em relação aos pais. A comparação entre as escolaridades médias dos pais e das mães, denuncia que elas não são significativamente diferentes. Nota-se também que há uma maior incidência de migrantes e de trabalhadores carteira assinada.

Com relação ao restante das informações, observa-se que há uma predominância de indivíduos brancos e residindo nas áreas mais dinâmicas do país. No que se refere à ocupação dos pais, cerca de $86 \%$ trabalham em setores intensivos em baixo estoque de capital humano.

\section{Resultados}

A captação do nível de desigualdade de oportunidades requer a comparação entre as distribuições para a renda observada e simulada. Para tanto, serão adotados dois procedimentos: no primeiro, abordagem indireta, os efeitos das variáveis de circunstância serão apagados; no segundo, abordagem direta, apagam-se os diferenciais de esforço. Em cada procedimento, estimam-se as distribuições condicionadas do esforço, totalizando três inferências, dado que se consideram três variáveis responsibility (anos de estudo, migração e o status no mercado de trabalho). No segundo passo, obtem-se a distribuição condicionada de renda, chegando, por fim, na distribuição incondicionada dos rendimentos (Eq. 3).

Os detalhes relativos aos procedimentos iniciais, em especial, a especificações das funções de regressão e o cálculos das distribuições condicionadas do esforço e da renda, serão abordados na subseção 4.1. Em seguida, discutemse os resultados relacionados a captação da desigualdade de oportunidades brasileira (subseção 4.2 ).

\footnotetext{
${ }^{13}$ Seguindo a mesma classificação de Bourguignon et al. (2007).

${ }^{14}$ Categorias: 1) Baixo Inferior: Lavrador, pescador, lenhador, etc; 2) Baixo Superior: braçal, servente, vigias, etc.; 3) Médio Inferior: pedreiro, eletricista, marceneiro, etc.; 4) Médio Médio: pracistas, viajantes, chefes de nível baixo, etc.; 5) Médio Superior: assessores, gerentes de alto nível, diretores, etc. e; 6) Alto: grandes proprietários, magistrados, nível superior, etc.

${ }^{15} \mathrm{Se}$ o indivíduo reside em um cidade diferente do seu local de nascimento.
} 
Tabela 1: Estatísticas Descritivas

\begin{tabular}{|c|c|}
\hline \multicolumn{2}{|l|}{ Variáveis } \\
\hline Média dos Rendimentos & $1.655,36$ \\
\hline \multicolumn{2}{|l|}{ Variáveis de Esforço } \\
\hline Média da Escolaridade & 6,97 \\
\hline Migrantes \% & $64 \%$ \\
\hline \multicolumn{2}{|l|}{ Status no Mercado de Trabalho \% } \\
\hline Formal & $57 \%$ \\
\hline Informal & $14 \%$ \\
\hline Conta-própria & $29 \%$ \\
\hline \multicolumn{2}{|c|}{ Variáveis de Circunstâncias } \\
\hline Média Esc. do Pai & 3,06 \\
\hline Média Esc. da Mãe & 2,99 \\
\hline \multicolumn{2}{|l|}{ Raça } \\
\hline Branco & $61 \%$ \\
\hline Não-branco & $39 \%$ \\
\hline \multicolumn{2}{|l|}{ Regiões } \\
\hline Norte \& Nordeste & $31 \%$ \\
\hline Sul, Sudeste e Centro-oeste & $69 \%$ \\
\hline \multicolumn{2}{|l|}{ Ocupação dos Pais } \\
\hline Baixo Inferior & $26 \%$ \\
\hline Baixo Superior & $34 \%$ \\
\hline Médio Inferior & $26 \%$ \\
\hline Médio Médio & $8 \%$ \\
\hline Médio Superior & $4 \%$ \\
\hline Alto & $2 \%$ \\
\hline
\end{tabular}

\subsection{Análise Preliminar}

Como já destacado na subseção 3.2, as estimativas para as distribuições contrafactuais considerarão uma estrutura de regressão, descrita em 13. A equação 13 será inferida considerando uma especificação logit para a link function, $\Lambda^{16}$, e uma estrutura linear para $m(y, x)$. Para cada contrafactual, serão considerados 999 quantis (sendo o menor 0.001 e o maior 0.999, com intervalos de 0.001). Cada vetor de variáveis contrafactuais é construído considerando um termo aleatório simulado. Ou seja, para gerar o contrafactual do grupo de referência $j=1$, tendo como base as covariáveis de $j=0$, geram-se $U_{1}^{0} \sim U(0,1)^{17}$.

É importante salientar, que para cada estimação contrafactual é fixado um grupo de referência. No caso, das variáveis não dicotômicas, como educação dos filhos, pais e mães e a ocupação do pai, as referências serão os seus valores médios. Quando elas forem binárias, um dos grupos será a referência, por exemplo, os migrantes, os brancos e a região mais dinâmica.

Entretanto, tanto os métodos "indireto", quanto o "direto" dependem de uma etapa preliminar, onde são estimadas as distribuições condicionadas do esforço. Neste caso, serão três processos de inferência, mudando apenas a va-

\footnotetext{
${ }^{16}$ Consideraram-se outros estimadores, como o probit e de regressão quantílica. Porém, não houve uma mudança expressiva nos resultados. Os resultados omitidos podem ser solicitados junto ao autor correspondente por email.

${ }^{17}$ Este procedimento faz-se necessário uma vez que a geração de contrafactuais utilizando somente os valores previstos na regressão, corresponde a zerar as variâncias de todas as variáveis não observadas. Maiores detalhes podem ser obtidos em Bourguignon et al. (2008).
} 
riável dependente, isto é: $E_{i}$ ( $i=$ Anos de estudo, migração, status no mercado de trabalho) será uma função da idade, dummies para a raça, região e coorte, educação do pai e da mãe e ocupação do pai. Uma das estimativas para $m(y, x)$, usando os anos de estudo como variável dependente está disposta na Tabela $2^{18}$.

Tabela 2: Distribuição Condicionada da Educação

\begin{tabular}{|c|c|c|}
\hline \multirow[t]{2}{*}{ Variáveis } & \multicolumn{2}{|c|}{ MQO } \\
\hline & Coeficiente & Desvio Padrão \\
\hline$\overline{\text { Idade }}$ & -0.0133 & 0.0159 \\
\hline Educ. Pai & $0.3971 *$ & 0.0093 \\
\hline Educ. Mãe & $0.2145 *$ & 0.0089 \\
\hline Raça & $-1.1950 *$ & 0.0504 \\
\hline Regiões & $0.3661 *$ & 0.0536 \\
\hline \multicolumn{3}{|c|}{ Dumies de Ocupação do pai (Ref.: Baixo Inferior) } \\
\hline Baixo Superior & $0.6356 *$ & 0.0872 \\
\hline Médio Inferior & $2.6670 *$ & 0.0978 \\
\hline Médio Médio & $2.9048 *$ & 0.1765 \\
\hline Médio Superior & $2.6700 *$ & 0.1665 \\
\hline Alto & $2.3671 *$ & 0.2134 \\
\hline \multicolumn{3}{|c|}{ Dumies Para Coorte (Ref.: Coorte $C_{7}$ ) } \\
\hline$C_{1}$ & $-1.8909 *$ & 0.5705 \\
\hline$C_{2}$ & $-1.2444 *$ & 0.5532 \\
\hline$C_{3}$ & $-0.5116 * * *$ & 0.3468 \\
\hline$C_{4}$ & 0.0246 & 0.3439 \\
\hline$C_{5}$ & 0.0321 & 0.2745 \\
\hline$C_{6}$ & $0.2200 *$ & 0.0954 \\
\hline
\end{tabular}

Grosso modo, os resultados indicam que: a) há uma relação positiva e estatisticamente significativa entre os níveis educacionais do pais e a educação dos filhos; b) a ocupação do pai influencia positivamente no estoque de educação dos filhos; c) os não brancos apresentam um nível educacional mais baixo em relação aos brancos (brancos e asiáticos); c) as pessoas que residem nas regiões mais dinâmicas, Sul, Sudeste e Centro-oeste, têm um nível educacional mais alto do que as que residem nas regiões Norte e Nordeste.

Diante disso, como já destacado em estudos pregressos, entre eles, Ferreira \& Veloso (2003, 2006), Bourguignon et al. (2007), pôde-se confirmar que as heterogeneidades regionais e raciais, bem como os fatores relacionados à mobilidade intergeracional, são relevantes para a decisão de investir em capital humano no Brasil.

Na segunda etapa do procedimento, infere-se a distribuição condicionada da renda. Para tanto, considera-se o logaritmo da renda como variável dependente mantendo a estrutura de uma estimação da equação de salários minceriana. A diferença é que serão inseridas as variáveis de esforço geradas no passo anterior e respeitadas as particularidades das abordagens indireta e direta.

\footnotetext{
${ }^{18}$ Alerta-se para o fato de que o contrafactual não é gerando utilizando, exclusivamente, os valores contidos nesta regressão. A relação linear, $m(y, x)$, é apenas uma parte do processo. Como já ressaltado, ainda é necessário considerar a link function $\Lambda$.
} 
No caso da abordagem indireta, fixam-se as variáveis de circunstância, obtendo um nível de esforço simulado. Em seguida, estima-se a distribuição condicionada para a renda, considerando o esforço simulado e um nível fixo para as circunstâncias. Nessa etapa será possível isolar o efeito de cada variável de circunstância sobre o indicador de desigualdade. Por conta disso, serão calculados seis índices de desigualdade. Já a abordagem direta fixa o nível de esforço e observa a diferença oriunda das circunstâncias, resultando, portanto, em um índice de desigualdade.

Um exemplo da geração de um contrafactual é apresentado na Tabela 3. Nela têm-se os resultados para as distribuições do logaritmo da renda (fórmula 9) observada (“Obs.") e simuladas, com efeitos indiretos ("Ind.") e diretos ("Dir."), em quantis selecionados. No caso do método indireto, as variáveis de circunstância foram fixadas em seus valores médios.

Assim como esperado, as simulações diminuiram a dispersão na distribuição da renda. Isto é, houve uma redução nos desvios padrões das distribuições ("Desv.Pad."), indicando que os métodos contrafactuais, sejam diretos ou indiretos, tornaram a distribuição mais compacta. Sendo que, quando se apagam o efeitos das circunstâncias, "Ind.", há uma redução de forma mais pronunciada nos desvios padrões.

Tabela 3: Distribuições Observada e Simulada para o Log da Renda

\begin{tabular}{lccc}
\hline \multicolumn{4}{c}{ Distribuições } \\
\hline Quantis & Obs. & Ind. & Dir. \\
\hline .10 & 4.00 & 5.00 & 3.66 \\
.20 & 4.39 & 5.05 & 4.35 \\
.50 & 5.22 & 5.15 & 5.24 \\
.80 & 5.98 & 6.01 & 5.61 \\
.90 & 6.40 & 6.39 & 6.06 \\
Desv.Pad. & 0.95 & 0.90 & 0.92 \\
\hline
\end{tabular}

A observação dos quantis selecionados, indica que a equalização das circunstâncias promove um impacto mais expressivo na parte de baixo da distribuição, i.e., considerando o quantil 0.10 , o valor relativo ao efeito indireto é sempre superior ao da distribuição observada e, também, em relação a simulação do efeito direto. Já no topo da distribuição, a equalização do esforço (efeito direto) é mais incisiva do que o da equalização das circunstâncias.

\section{2 Índices de Desigualdade de Oportunidades}

Nesta subseção serão apresentados os resultados relativos aos contrafactuais 5 e 6. Serão calculados alguns funcionais, tais como, a curva de Lorenz e os indicadores de desigualdade que, no caso desse estudo, serão representados pelo índice de Theil ${ }^{19}$. As Figuras 1 e 2 sintetizam as informações para duas

\footnotetext{
${ }^{19}$ Convém ressaltar que há uma discussão relacionada à aplicação de um índice de desigualdade sobre uma variável obtida a partir de um processo contrafactual. Para detalhes, ver Devooght (2008). No mais, a decomposição da desigualdade de oportunidades nos seus efeitos direto e indireto, poderiam ser substituídas por medidas baseadas na "path independence". Essa alternativa é fornecida por Ferreira \& Gignoux (2011).
} 
simulações a partir das curvas de Lorenz. No primeiro caso, Figura 1, têmse a curva de Lorenz para a renda observada e contrafactual ${ }^{20}$. A geração do contrafactual se deu a partir do método indireto, com a equalização das circunstâncias.

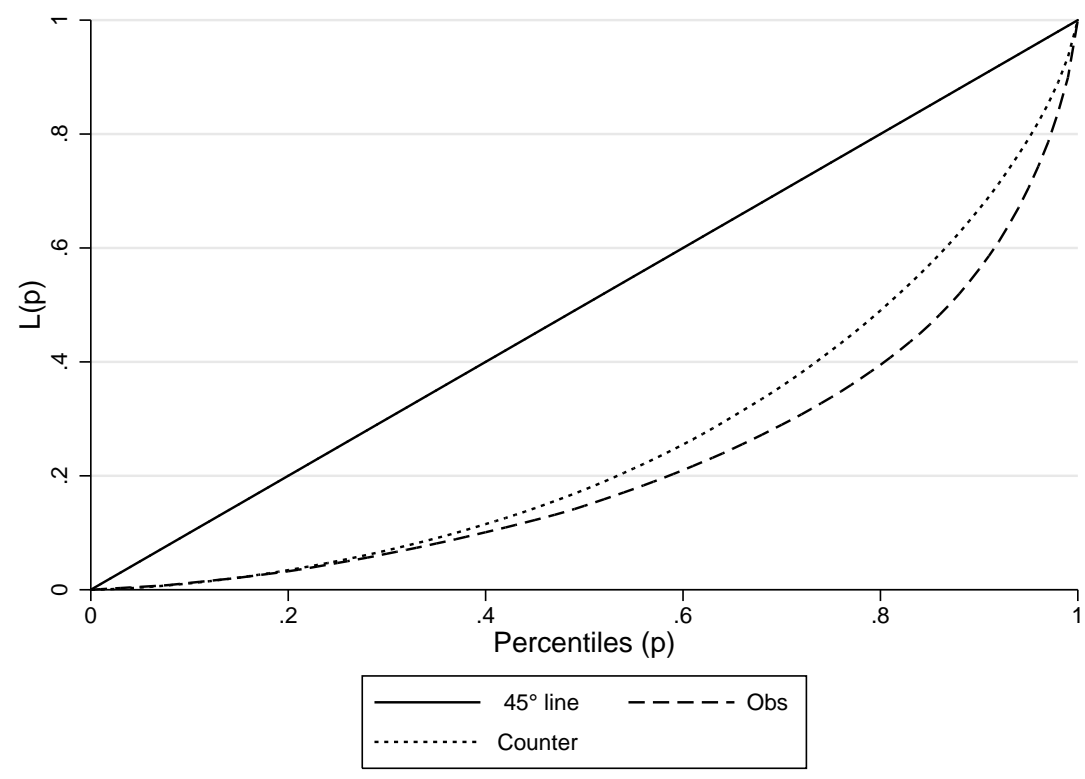

Figura 1: Curva de Lorenz para as Rendas Observada e Contrafactual: Método Indireto

Note que há uma contração na curva de Lorenz, ou seja, caso todos os indíduos tivessem acesso ao mesmo conjunto de oportunidades, o nível de desigualdade de renda seria inferior ao vigente. Contração similar é observada quando se utiliza o método direto, ou seja, apagando o efeito do esforço. Isso pode ser constatado a partir da Figura 2 que traz as curvas de Lorenz após a equalização do esforço (abordagem direta). Aparentemente há uma maior retração na curva de Lorenz, nesse último caso. Contudo, para se ter uma idéia do impacto desses fatores sobre a distribuição da renda, é necessário calcular os índices de desigualdade.

A Tabela 3 sintetiza os índices de Theil inferidos com base na abordagem indireta, onde serão fixados os fatores de circunstância, um a um. É importante salientar que a desigualdade total da renda é de 0,5639. Diante disso, a Tabela 3 informa que após a equalização de todos os fatores de circunstância (Total), a desigualdade cai para 0,3689. Ou seja, a contribuição da desigualdade de oportunidades para a desigualdade de renda é de cerca de 35\%. Quando fixados um a um, observa-se que o fator "educação do pai" é o que mais contribui para a desigualdade de oportunidades.

Em linhas gerais, esses resultados são similares aos obtidos por Bourguignon et al. (2007), como, por exemplo, o que destaca a importância do backgroud familiar para a determinação do nível de oportunidades. Contudo, os

\footnotetext{
${ }^{20}$ Os resultados dessa seção baseiam-se na aplicação do exponencial sobre os contrafactuais, dado que na especificação da regressão, utiliza-se o log da renda.
} 


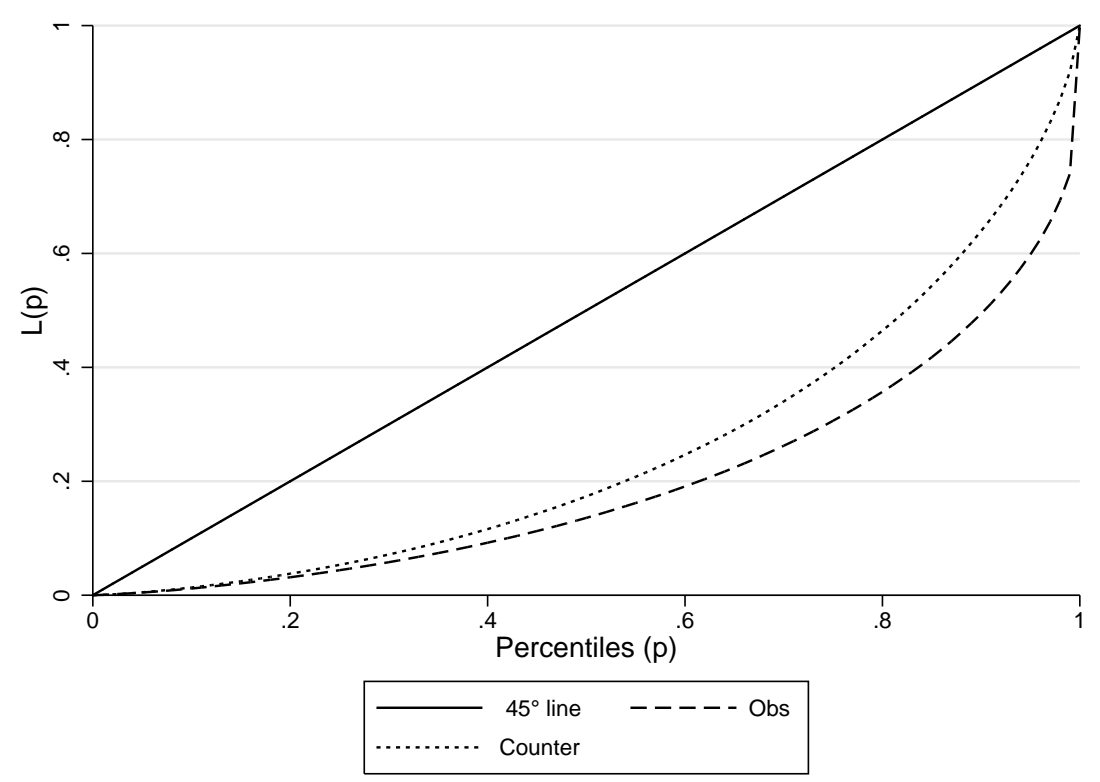

Figura 2: Curva de Lorenz para as Rendas Observada e Contrafactual: Método Direto

Tabela 4: Índice e contribuição da $I O_{P}$ Brasileira: Abordagem Indireta

\begin{tabular}{lcccccc}
\hline & Total & Raça & Região & Educ. Pai & Educ. Mãe & Ocup. Pai \\
\hline Theil & 0,3689 & 0,4019 & 0,3967 & 0,3864 & 0,4001 & 0,3929 \\
\hline Total & Raça & Região & Educ. Pai & Educ. Mãe & Ocup. Pai \\
\hline Contribuição & $35 \%$ & $29 \%$ & $30 \%$ & $31 \%$ & $29 \%$ & $30 \%$ \\
\hline
\end{tabular}

autores apontam para um impacto da desigualdade de oportunidades sobre a desigualdade total de cerca de $23 \%$, valor bem inferior aos $35 \%$ da Tabela 3 .

Para confirmar esse resultado, foi estimado o índice de desigualdade de oportunidades a partir do método direto. Ou seja, apagando os diferenciais de esforço e observando a desigualdade resultante apenas das circunstâncias. Nesse caso, o índice de Theil encontrado foi de 0,3721, o que corresponde a um impacto de cerca de $34 \%$ sobre a desigualdade total.

\section{Considerações Finais}

Esse artigo utilizou a proposta teórica contida Pistolesi (2009) para determinar a desigualdade de oportunidades brasileira utilizando como base os dados da PNAD 1996. Para isso, considerou as críticas presentes na literatura à abordagem semi paramétrica do Donald et al. (2000) e empregou o método de geração dos contrafactuais apresentado por Chernozhukov et al. (2009). Trata-se de uma estratégia mais ampla que permite uma melhor captação dos efeitos das covariáveis a partir da utilização de estimadores que possuem distribuição assintótica bem definida. Os resultados indicam que a proporção da desigualdade de oportunidades em relação à desigualdade total é de certa 
de $35 \%$, share bastante superior aos $23 \%$ encontrado por Bourguignon et al. (2007).

\section{Agradecimentos}

O primeiro Autor gostaria de agradecer o suporte financeiro do $\mathrm{CNPq}$ - projeto $475225 / 2009-0$.

\section{Referências Bibliográficas}

Angrist, J. \& Pischke, J. (2008), Mostly Harmless Econometrics: an Empiricists Companion, Princeton Univesity Press, Princeton.

Atkinson, A. (1970), 'On the measurement of inequality', Journal of Economic Theory 2(3), 244-263.

Azevedo, J. (2007), Avaliando a significância estatística da queda na desigualdade no Brasil. In: Barros, R., Foguel, M.E Ullysea, G. (Orgs). Desigualdade de renda no Brasil : uma análise da queda recente, IPEA, Brasilia.

Barros, R., Ferreira, F., Vega, J., Chanduvi, J., Carvalho, M., Franco, S., FreijeRodríguez, S. \& Gignoux, J. (2009), Measuring inequality of opportunities in Latin America and the Caribbean, World Bank, Washington.

Bourguignon, F., Ferreira, F. \& Leite, P. (2008), 'Beyond oaxaca-blinder: Accounting for differences in household income distributions', Journal of Economic Inequality 6(2), 117-148.

Bourguignon, F., Ferreira, F. \& Menéndez, M. (2007), 'Inequality of opportunity in brazil', Review of Income and Wealth 53, 585-618.

Checchi, D. \& Peragine, V. (2009), 'Regional disparities and inequality of pportunity: the case of italy', Journal of Economic Inequality, forthcoming .

Chernozhukov, V., Fernández-Val \& Melly, B. (2009), 'Inference on counterfactual distributions', CEMMAP Working Papers CWP09/09.

Cogneau, D. \& Gignoux, J. (2005), 'Earnings inequalities and educational mobility in brazil over two decades', Développement, Institutions \& Analyses de Long terme DT/2005/03.

Devooght, K. (2008), 'To each the same and to each his own: a proposal to measure responsibility-sensitive income inequality', Economica 75, 280-295.

DiNardo, J., Fortin, N. \& Lemieux, T. (1996), 'Labor market institutions and the distribution of wages, 1973-1992: a semiparametric approach', Econometrica 64, 1001-1044.

Donald, S., Green, A. \& Paarsch, H. (2000), 'Differences in wage distributions between canada and the united states: an application of a flexible estimator of distribution functions in the presence of covariates', Review of Economic Studies 67, 609-633. 
Ferreira, F. \& Gignoux, J. (2011), 'The measurement of inequality of opportunity: Theory and an application to latin america', Review of Income and Wealth 57(4), 622-657.

Ferreira, S. \& Veloso, F. (2003), 'Mobilidade intergeracional de educação no brasil.', Pesquisa e Planejamento Econômico 33, 481-583.

Ferreira, S. \& Veloso, F. (2006), 'Intergenerational mobility of wages in brazil', Brazilian Review of Econometrics 26, 181-211.

Figueiredo, E. \& Ziegelmann, F. (2009), 'Mudança na distribuição de renda brasileira: Significância estatística e bem-estar econômico', Revista de Economia Aplicada 13, 257-277.

Figueiredo, E. \& Ziegelmann, F. (2010a), 'Estimating income mobility using census data', Physica A: Statistical Mechanics and its Applications 389(21), 4897-4903.

Figueiredo, E. \& Ziegelmann, F. (2010b), 'Estimation of opportunity inequality in brazil using nonparametric local logistic regression', Journal of Development Studies 46(9), 1593-1606.

Firpo, S., Fortin, N. \& Lemieux, T. (2007), 'Unconditional quantile regressions', Econometrica, forthcoming .

Giustinelli, P. (2010), 'Non-parametric bounds on quantiles under monotonicity assumptions: with an application to the italian education returns.', Journal of Applied Econometrics .

Gosling, A., Machin, S. \& Meghir, C. (2000), 'The changing distribution of male wages in the u.k.', Review of Economic Studies 67, 635-666.

Han, A. \& Hausman, J. (1990), 'Flexible parametric estimation of duration and competing risk models', Journal of Applied Econometrics 5, 1-28.

IPEA (2006), 'Sobre a recente queda na desigualdade de renda no brasil', Nota Técnica IPEA.

Kranich, L. (1996), 'Equitable opportunities: an axiomatic approach', Journal of Economic Theory 71, 132-147.

Lefranc, A., Pistolesi, N. \& Trannoy, A. (2004), 'Harder times for heirs: Social background and income in france', Mimeo THEMA pp. 1979-2000.

Lefranc, A., Pistolesi, N. \& Trannoy, A. (2008), 'Inequality of opportunities vs. inequality of outcomes: are western societies all alike?', Review of Income and Wealth 54, 513-546.

Machado, J. \& Mata, J. (2000), 'Counterfactual decomposition of changes in wage distributions using quantile regression', Journal of Applied Econometrics 68(4), 997-1012.

Manski, C. \& Pepper, J. (2000), 'Monotone instrumental variables, with an application to the returns to schooling', Econometrica 20, 445-465. 
O’Neil, B., Sweetman, D. \& Van de Gaer, D. (2001), Equality of opportunity and kernel density estimation: an application to intergenerational mobility. In: Fomby, T. E Hill, C. (eds.). Applying kernel and nonparametric estimation to economic topics. Advances in Econometrics, Vol. 14, JAI Press, Stanford.

Ooghe, E., Schokkaert, E. \& Van de Gaer, D. (2007), 'Equality of opportunity versus equality of opportunity sets', Social Choice and Welfare 28, 209-230.

Pastore, J. \& Silva, N. (1999), Mobilidade Social no Brasil, Makron Books, São Paulo.

Pattanaik, P. \& Xu, Y. (1990), 'On ranking opportunity sets in terms of freedom of choice', Recherches Economiques de Louvain 56, 383-390.

Pistolesi, N. (2009), 'Inequality of opportunity in the land of opportunities', Journal of Economic Inequality 7, 411-433.

Ramos, C. \& Santana, R. (2003), 'Quão pobres são os pobres?', UNB: Texto para Discussão pp. 1991-2001. Brasil.

Ramos, X. \& Van de gaer, D. (2009), 'Empirical evidence on inequality of opportunity', Working Paper .

Roemer, J. (1996), Theories of Distributive Justice, Harvard University Press, Cambridge.

Roemer, J. (1998), Equality of Opportunity, Harvard University Press, New York.

Van der Vaart, A. \& Wellner, J. (1996), Weak Convergence and Empirical Processes: with Applications to Statistics, Springer, New York. 